\title{
Steganography by Multipoint Arabic Letters
}

\author{
Ammar Odeh, Aladdin Alzubi, Qassim Bani Hani, Khaled Elleithy \\ Department of Computer Science \& Engineering, University of Bridgeport \\ Bridgeport, CT 06604, USA
}

\section{Abstract}

Security issues are taken into consideration for many applications, where transfering sensitive data over network must be protected from any man-in-the-middle attackers. proivacy of data can be granted using encryption, by changing transmitted data into cipher form. Apart from encryption, hiding of data represents another technique to transfer data without being noticble by an attacker which is called Steganography. In this paper, we will discuss the main concepts of Steganography and a carrier media that is used for this goal.

\section{Introduction}

Steganography is a Greek words coming from cover text. Where "Stegano" means hidden and "Graptos" means writing. The secure data will be embedded into other object, so middle attacker can't catch it [1]. Invisible ink is an example for Steganography using a readable message transfer between source and destination. Everyone in the middle can read the message without having any clue about the hidden data .On other hand, authorized persons can read it depending on substances features [2][3].

Ancient Greeks used to shave the messenger head and then wait until hair grow back, then themessage will be sent to the destination [1]. Depending on this method we have 2 possibilities:-

Message arrived so the receiver can read the message and recognize if message is changeed or not.

If message did not arrive that mean the attacker detect the message.

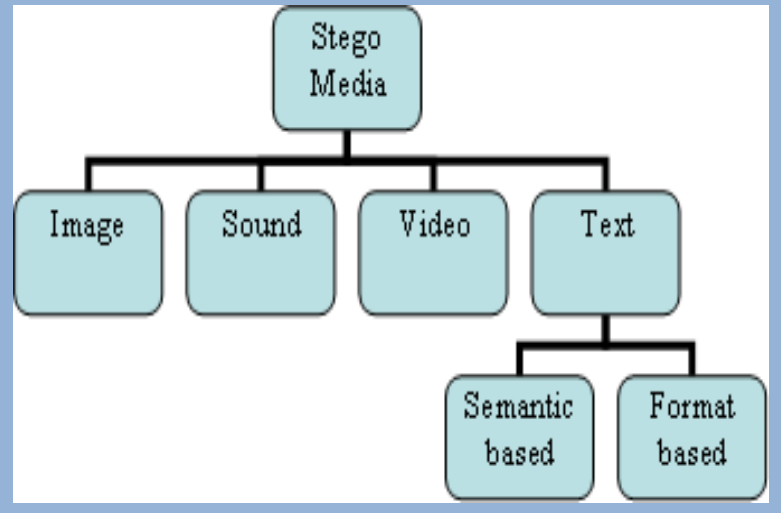

\section{Method}

Pointed letter represents one of characteristic in Arabic/Persian languages. Where English language does not have the same number only we can find (i, j).

Table I Arabic letters regards to number of point

\begin{tabular}{|c|c|}
\hline letter & Number of point \\
\hline 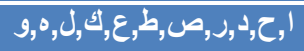 & 0 \\
\hline ب,ج,خ,ذ,ذ,j,ض,ظ,غ,غ,فرن & 1 \\
\hline ترقري & 2 \\
\hline$\stackrel{\sharp}{\sharp}$ & 3 \\
\hline
\end{tabular}

Our algorithm tries to hide data in multipoint Arabic/Persian letter like $(\uplus$, tha) where in Arabic language we have five letters, eight in Persian.

\section{Method (cont)}

Each character can hide 2 bits determine by shifting and distance between letter points.

\section{Result}

Table II Capacity of webpage for different Arabic website

\begin{tabular}{|r|c|c|c|c|}
\hline$\#$ & Page Name & $\begin{array}{c}\text { Page } \\
\text { Size }\end{array}$ & $\begin{array}{c}\text { Character \# } \\
\mathbf{2} \text { point or } \\
\text { more }\end{array}$ & $\begin{array}{c}\text { Capacity } \\
\text { Ratio } \\
\text { (Bit/ } \\
\text { Kilobyte) }\end{array}$ \\
\hline 1 & aljazeera.net & $\begin{array}{r}23.8 \\
\text { KB }\end{array}$ & 1245 & 105 \\
\hline 2 & daralhayat.com & $\begin{array}{r}15.4 \\
\text { KB }\end{array}$ & 968 & 126 \\
\hline 3 & salahws.com & $\begin{array}{r}10.3 \\
\text { KB }\end{array}$ & 535 & 104 \\
\hline 4 & holyquran.net/tadabur & $\begin{array}{r}13.8 \\
\text { KB }\end{array}$ & 516 & 75 \\
\hline 5 & khayma.com & $\begin{array}{r}21.8 \\
\text { KB }\end{array}$ & 499 & 46 \\
\hline
\end{tabular}

Table III Vertical Point shifting versus Multipoint algorithm

\begin{tabular}{|c|c|c|}
\hline \multicolumn{3}{|c|}{ 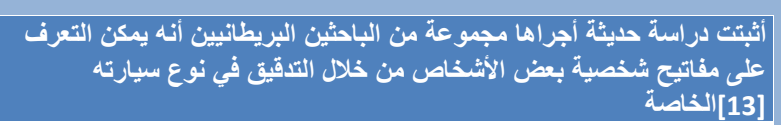 } \\
\hline & Number of letter & $\begin{array}{c}\text { Number of hidden } \\
\text { bit }\end{array}$ \\
\hline Pointed letter & 50 & 50 \\
\hline Multipoint letter & 29 & 58 \\
\hline
\end{tabular}

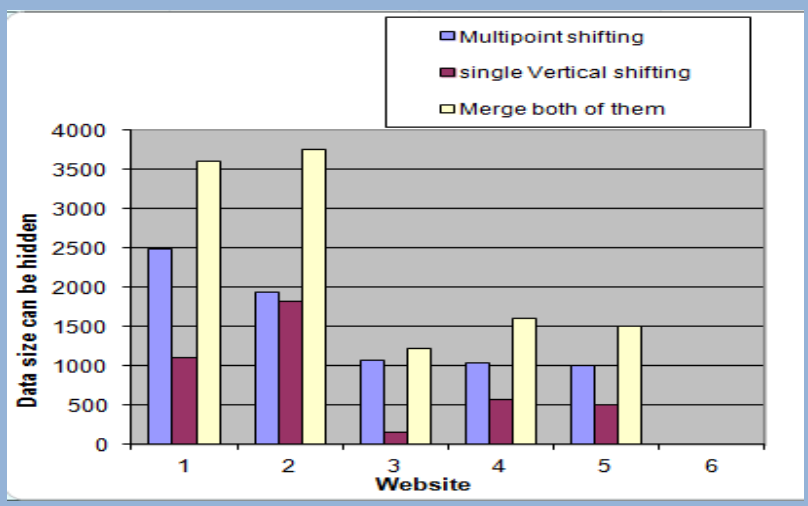

Figure 1. Marge between Vertical and multipoint algorithm

\section{Conclusion}

In our paper we introduced new text Steganograph in Arabic multipoint letters. Our algorithm deals with two bits for each multipoint letter,

\section{References}

[1] Aelphaeis Mangarae "Steganography FAQ," Zone-H.Org March 18th 2006 [2] S. Dickman, "An Overview of Steganography," July 2007.

[3] V. Potdar, E. Chang. "Visibly Invisible: Ciphertext as a Steganographic Carrier," Proceedings of the 4th International Network Conference (INC2004), page(s):385-391, Plymouth, U.K., July 6-9, 2004 